\title{
APROXIMACION AL CONCEPTO DE ESCRITURA EN GEORGES PEREC
}

\author{
Jesús Camarero Arribas* \\ Universidad del País Vasco
}

\author{
RESUMEN
}

El enfoque de un análisis a realizar sobre la obra de un autor puede instalarse en el terreno de las Formas. Pero dentro de este ámbito hay posibilidad de delimitar aún más perfectamente dicho enfoque, escogiendo las pautas concretas prescritas por un régimen muy particular: puede tratarse del enfoque lingüistico (cuyo objeto sería la lengua, estrictamente), el análisis del género al que pertenece dicha obra o, en último lugar, tener en cuenta la dimensión puramente escritural del fenómeno literario.

La escritura, como ciencia, no tiene aún delimitado su campo definicional, a pesar de haber sido ya varios los intentos realizados en ese sentido (ver la obra de Derrida o el grupo Tel Quel). Por ello, todo ensayo de análisis que utilice como método los principios de la "estética» escritural, ha de tener como fin-además del objetivo critico de todo análisis- el aportar nuevas experiencias en este campo nuevo que se revela fecundo en extremo.

Al tener como meta la elaboración de un concepto primario de lo escritural perequiano, hemos pretendido fundamentalmente poner al descubierto lo que constituye un auténtico haz de elementos constitutivos y factores determinantes que, unidos, conforman lo que podríamos denominar el "recorrido escritural» del autor. Desde la génesis de la obra. W ou le souvenir d'enfance, hasta la construcción de un microcosmos imaginario de grandes dimensiones, La Vie mode d'emploi, se puede detectar un largo itinerario de aprendizaje escritural del autor que, por otra parte, se preocupó «cuidadosamente» de dejar constancia clara de ello en sus obras. Este fenómeno interesantísimo de la puesta en evidencia de los mecanismos de escritura, constituye nuestra principal fuente de análisis y nos permite instrumentalizar el proceso que habrá de conducirnos al concepto perseguido.

Este conjunto de resortes que articulan el mecanismo productor de escritura en Perec son, finalmente, la clave que explica la evolución de la literatura en los últimos tiempos. Ellos son la puesta en evidencia, de nuevo, del modo de hacer de los nuevos escritores más preocupados quizá por el funcionamiento de los medios a su disposición que por un mensaje universal y utopico.

* Dr. en Filología Francesa. Dpto. de Filología Francesa, Facultad de Filología, Universidad del País Vasco, Marqués de Urquijo, s/n; 01006 Vitoria-Gasteiz. Recibido el 11-9-1987. 


\title{
RESUME
}

La façon d'envisager l'analyse de l'oeuvre d'un écrivain peut se faire dans le domaine des Formes. Mais il y a la possibilité plus concrète et parfaite de déterminer le soi-disant projet: en choisir les règles prévues par un régime très particulier. Il pourrait être l'optique linguistique (dont l'objet serait la langue, strictement) l'analyse du genre auquel apartient l'oeuvre ou, enfin, envisager la dimension purement scripturale du phénomène littéraire.

Malgré plusieurs essais réalisés principalement par Derrida ou le groupe Tel Quel, l'écriture, comme science, n'a pas encore fixé son champ définitionnel. C'est à cause de cela que toute expérience portant sur la méthode des principes de l'resthétique» scripturale, doit avoir comme but -outre l'objectif critique de toute analysel'apport de nouveaux résultats dans ce champ qui relève d'une fécondité extrême.

Puisque nous avions comme but l'élaboration d'un concept primaire du scriptural péréquien, nous avons poursuivi fondamentalement une mise au point de ce qui constitue le faisceau des éléments constitutifs et des facteurs déterminants qui, unis, conforment ce que nous pourrions nommer le «parcours scriptural» de l'auteur. Depuis la génèse de l'oeuvre. W ou le souvenir d'enfance, jusqu'à la construction d'un microcosme imaginaire de haute performance, La Vie mode d'emploi, on peut facilement constater un long itinéraire d'apprentissage scriptural de la part de l'auteur qui, d'ailleurs, a eu le souci d'en laisser les traces. Ce phénomène très intéressant de la mise en évidence des mécanismes d'écriture, constitue notre source principale pour l'analyse et il nous permet d'instrumenter le processus qui devra nous conduire vers le concept désiré.

L'ensemble des ressorts qui articulent le mécanisme producteur de l'écriture chez Perec sont, enfin, la clef qui explique l'évolution de la littérature de nous jours. Ils sont aussi la mise en évidence du modus faciendi des écrivains nouveaux, peut-être plus préoccupés par le fonctionnement des moyens mis à leur disposition que par un message universel et utopique.

\begin{abstract}
Le moment apparaît toujours dans la vie d'un écrivain où, son génie aidant, l'envie lui prend de faire craquer sa langue. Toute littérature est une bataille sur les frontières de la langue, une lutte pour, avec des mots. tenter de les dépasser, ou, si l'on veut, faire entrer dans sa langue ce qui se refuse à y pénétrer.
\end{abstract}

J. PEIGNOT, De l'écriture à la trpographic'

El escritor utiliza un código determinado de signos dentro de una lengua, habla una lengua concreta (razón antropológica). La lengua es el elemento «utilizado» para escribir: la escritura de la obra sufre el determinismo del lenguaje en cuanto que no puede optar por una lengua, sino que la lengua le viene impuesta.

El escritor tiene un estilo determinado, dependiendo de sus características como escritor y de una fórmula estética propia de su obra (razón estética). El estilo, además, puede sufrir modificaciones en la diacronía de la misma obra, pues puede contemplar fórmulas distintas dentro de la consideración general de un estilo concreto.

La forma, como aglutinante de la lengua y el estilo, es una categoría que identifica al escritor. Sin embargo, si la pertenencia a una comunidad lingüistica y la patente de un estilo propio parecen afirmar la identidad del escritor y su pertenencia a un grupo social, también es cierto que ambos factores (la lengua, el estilo) pueden llegar a condicionar y limitar gravemente la tarea del escritor. 
En primer lugar, la lengua supone una barrera, «une ligne dont la transgression désignera peut-être une surnature du langage»' ${ }^{\prime}$, como estereotipo comunicativo del grupo social supone un molde irrompible que puede ser utilizado como motivo para la creación.

En segundo lugar, si es verdad que el estilo es lo más arbitrario y menos domesticable, reacio a los condicionamientos de cualquier regulación o lógica impuesta, sin embargo «il est le produit d'une poussée, non d'une intention, il est comme une dimension verticale et solitaire de la pensées ${ }^{2}$, lo cual supone una patente del tipo de escritor que imprime un carácter indefectible e imborrable.

De alguna manera parece entonces que hay una consideración de la lengua y el estilo que no es positiva cara a la actividad del escritor. No pueden ser transformados, son rígidos y carecen de esa elasticidad propia de las formas.

La escritura es la tercera dimensión de la forma. La diferencia y la novedad residen en ese concepto de "acte de solidarité historique» ${ }^{3}$ que supone superar ampliamente las limitaciones ya vistas en la lengua y en el estilo. El acto de solidaridad histórica es el rasgo fundamental para entender la superación de esos niveles lingüísticos y estilísticos en los que está ausente: en la lengua, porque viene impuesta y puede deducirse cierta impotencia del escritor ante esta limitación constrictiva; en el estilo, porque es algo personal e intransferible. En ambos casos se produce una intranscendencia en lo inmediato que impide la agilización del proceso escritura-lectura. Este destino social de la literatura a través de la escritura remite de nuevo al escritor a sus fuentes. Escribiendo, parte de la escritura y llega a la escritura al mismo tiempo que la literatura se afirma de continuo ${ }^{4}$.

La escritura (como labor artesanal) es definitivamente un oficio, un trabajo o una ocupación y hay para ello las razones siguientes:

1. ${ }^{\circ}$ por la responsabilidad del acto creador: el acto de escribir, de producir escribiendo y de producir escritura, es trascendental porque el escritor es consciente de que la obra se integra de lleno en ese destino social de la escritura; por el acto mismo de la creación libre en correspondencia con la integración del escritor en la sociedad a través de su obra;

$2 .^{\circ}$ porque el escritor ofrece su trabajo, su esfuerzo en escribir la obra (no sólo la obra misma, producto final) y, al margen de cualquiera otra consideración, la obra se presenta como elaboración, como el proceso mismo de su construcción.

De alguna manera estamos ante el escritor total. Aquel que no sólo entrega la obra al lector, sino también hace participar, a través de la relación de/por la escritura, del proceso de construcción de la obra misma.

La definición de Georges Perec escritor comporta dos rasgos significativos por el contenido y la trascendencia que tienen: el oulipismo y el hecho de escribir ${ }^{5}$. De ellos es el segundo el que aquí nos interesa, por esa relación que se establece a los largo de toda la obra del autor entre el oficio y la pasión, lo cotidiano y la vocación, los libros y los libros. El producto de la actividad escritural es la literatura como conjunto más abstracto (irreal) donde esta actividad se transforma y se compromete con el rol social que hace

1. BARTHES, R., Le degré zéro de l'écriture, Seuil, París, 1953, 1972, p. 11.

2. Ib., p. 12.

3. Ib., p. 14.

4. «La littérature commence avec l'écriture. (...) ce qui est écrit appartient à la littérature, (...) celui qui le lit lit de la littérature», BLANCHOT, M., Le livre à venir, Gallimard, París, 1959, p. 302.

5. Para el primero de ellos se puede encontrar una definición general con múltiples ejemplos del autor en mi artículo: «El arquetipo de la constricción oulipiana» in Sobre literatura potencial, Univ. País Vasco, Zaragoza, 1987, p. 49-92. 
posible el fenómeno literario en toda su dimensión. Cuando el escritor se interesa por el proceso de su propia escritura y de ello resulta una reflexión consciente y profunda, este escritor está en condiciones de añadir a su obra una trascendencia aún mayor y, en consecuencia, de abrir paso a la posibilidad de un cambio en la expresión escritural y literaria de dicha obra. Desde el momento en que el escritor trata de responder a las preguntas por siempre planteadas y si se producen respuestas que interesan directamente al porvenir de su escritura, esta actitud reflexiva relacionada con su trabajo nos presenta un escritor nuevo y distinto: el escritor-escritor, aquel que proporciona la explicación del hecho de escribir al mismo tiempo que escribe, es decir, se escribe porque se había escrito (o leído) o porque estaba ya escrito (o leído). De esta manera se empieza a responder a la pregunta última de $\dot{c}$ a dónde va la literatura?, ese proyecto indeleble que es el objeto permanente de la crítica literaria. Será por tanto la propia escritura perequiana la que nos proporcione las diferentes claves que articulan un concepto nuevo y particular de lo escritural en Perec, como siempre extrapolable a otras experiencias de lo literario ${ }^{6}$.

La escritura se define por sus propios trazos, por sí misma, por la inmediatez y evidencia de sus componentes (puesto que tal es la función que ella misma se asigna); de modo que esos elementos conformadores que posibilitaron el hecho escritural son luego los delatores de la propia escritura a través de la puesta en evidencia de sí mismos, con lo que se puede decir que nada hay tan transparente como lo escritural. Y ello si tenemos en cuenta sobre todo el producto final al que indefectiblemente se llega, el texto.

El texto es algo «indéfinissable, exclusif, sans retour» ${ }^{7}$, por tanto experiencia única de un solo experimento escritural, de un intento en el que todo se halla incluido como experiencia (hablamos en el nivel puramente de la escritura). Por ello el texto nos ocupará ampliamente en esa vertiente por la que se constituye en el punto de inflexión de una experiencia única e irrepetible, el rasgo total y definitivo de lo que es la escritura.

El hecho también de hablar aquí de una nueva categoría de lo literario, la escritura, no es simplemente la constatación de que se ha producido un cambio o estamos imbuidos en un proceso alternativo, y no sólo esto, sino también que hemos llegado a un estadio de la producción literaria en el que esa comunicación clara y fecunda que se produce en/por la obra se da como fenómeno distinto. Algunos autores ya empiezan a hablar incluso de «contre-communication»", de intimidación, de una forma nueva de producir esa comunicación que, básicamente, funciona en el nivel escritor-lector, pero que bajo un criterio escritural hay que conducir al ámbito de lo estrictamente textual, puesto que en el texto es donde se producen -con esta nueva óptica- todos los efectos de sentido debidos a la escritura y analizables por criterios escriturales ${ }^{9}$. Pero todavía hay más: al decidir delimitar un nuevo campo de lo escritural y aislar de algún modo una nueva categoría, la escritura se constituye en una esencia distinta y ya no sirven algunos criterios que hasta ahora venían funcionando en el análisis literario.

6. El hecho de que hagamos referencia directa al binomio escritura-lectura viene justificado ampliamente por la fusión que puede llegar a alcanzarse de ambos actos, tal como lo demuestra esta cita: «Je lis peu, mais je relis sans cesse, Flaubert et Jules Verne, Roussel et Kafka, Leiris et Queneau; je relis les livres que j'aime et j'aime les livres que je relis, et chaque fois avec la même jouissance, que je relise vingt pages, trois chapitres ou le livre entier: celle d'une complicité, d'une connivence, ou plus encore, au-delà, celle d'une parenté enfin retrouvée» (G. Perec, $W$ ou le souvenir d'enfance, Denoël, París, 1975, p. 193; trad. esp.: $W$ o el recuerdo de la infancia, Península, Barcelona, 1987).

7. THIBAUDEAU, J., «Le roman comme autobiographie», Tel Quel, Théorie d'ensemble, Seuil, París, 1968, p. 198.

8. Barthes, op. cit., p. 18.

9. Este trabajo pretende mostrar también una vía de análisis siguiendo dichos criterios, a ella nos referiremos después. 
La escritura es una categoría fuertemente autonímica, «la literatura (...) sabe que escribiendo no se puede decir nada que sea exterior a la escritura, ninguna verdad que no sea una verdad referente al acto de escribir» ${ }^{10}$, es decir, nada que esté escrito escapa al carácter de lo escritural, aun a pesar de que otras vías son posibles. A través de la función-reflejo que es la autonimia, la escritura dejará constancia en el texto de todo aquello que responde a la pregunta de iqué es la literatura?: «écrire, c'est seulement écrire, tracer des lettres sur une feuille blanche»" ", los trazos del signo sobre la página sin mácula. La escritura es lo próximo, lo inmediato, lo rápidamente aprehensible y, casi podríamos decir que lo primero, si no fuera por esa relación íntima con la lengua en la función de leer-escribir que reduce el axioma a un círculo infinito. Es como si, precisamente, se hubiera entrado en la consideración de la lengua como amarre que no sostiene más que débilmente el funcionamiento de lo literario y que entonces hay que encontrar una fijación más sólida, sobre todo en una época como la nuestra en la que «l'absence de repères» ${ }^{12}$ es una característica de lo más definitoria. La ausencia de anclajes junto a la constatación del paso del tiempo inexorable, la afirmación de la obra como valor en sí junto a la perdurabilidad del scripta manent, son quizá las premisas que permiten formular la idea de un «encubrimiento» del tiempo en beneficio de la relevancia del espacio $^{13}$.

Al permanecer inmóvil, durable, la escritura no permite ningún filtro temporal, como si escribir fuera olvidar, alejar del proceso de la mente la permanencia de una idea, de lo cual ya no hay necesidad, puesto que el recuerdo -el tiempo- ha quedado en la escritura y se pone en evidencia por la lectura de su contenido.

Como todo fenómeno, la escritura nace vinculada a una serie de acontecimientos (no históricos en este caso). Basta simplemente operar con criterios plásticos para darse cuenta de que siempre se escribe sobre un soporte y que la escritura no sería posible sin esta determinación quasi «alienante» (por el contrario, el soporte permite la liberación expansiva de la escritura). Además, no hay que ignorar el peso específico que puede llegar a tener el soporte: su tamaño, color, peso, densidad, forma, relieve, etc., condicionan y complementan enormemente esa fusión con la escritura, en ese momento preciso en el que el signo se in-scribe en la página.

Así pues, el soporte de la escritura, la página básicamente, es una primera aproximación al carácter espacial de la escritura: en primer lugar, la página es un plano, una dimensión, un espacio (puede incluso llegar a ser un volumen), tal como ocurre en el libro sobre el espacio al señalarse: «essai sur la notion d'espace qui commence par celui de la page, puis passe du lit au monde entier» ${ }^{14}$; en segundo lugar, el modo espacial que adquiere la propia escritura en simbiosis con la página está en relación directa con lo anterior formando un concepto único:

10. CALVINO, I., «Dos entrevistas sobre ciencia y literatura», in Punto y aparte, Bruguera, Barcelona, 1983, p. 238.

11. PEREC, G., «Les lieux d'une ruse», Cause Commune, n. ${ }^{\circ}$ 1, U.G.E., París, 1977, p. 79.

12. PEREC, G., $W$ ou le souvenir d'enfance, p. 93-94.

13. BLANCHOT, M., L'espace littéraire, Gallimard, París, 1955.

14. MATHEWS, H., «Le catalogue d'une vie», Magazine littéraire, n. ${ }^{\circ}$ 193, 1983, p.16. 
feuille banche, noircit l'espace vierge, lui donne un sens, le vectorise: de gauche à droite

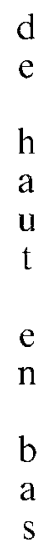

Desde aquí es fácil pasar a otras consideraciones concernientes al modo de funcionamiento de lo espacial en la obra, es decir, cómo se va constituyendo el espacio a medida que se construye la obra. Descendiendo al ámbito puramente oulipiano, la práctica de la constricción queda totalmente comprendida en este registro, pues así se constata en el funcionamiento de gran parte de los textos de Perec, como es el caso de Quel petit vélo à guidon chromé au fond de la cour ${ }^{\text {16 }}$, donde «la répétition de mots ou même de phrases formant à travers tout le livre une trame enjouée» ${ }^{17}$ es la perfecta descripción de esa red de correspondencias que se producen en el interior de la obra y que conforman el modo espacial ya referido. La distribución de esos componentes reiterados ordenada y sistemáticamente (sistémicamente) constituye ese espacio escritural que se denominará «escritura espacial», donde el texto -como ya se ha anticipado- es la referencia absoluta y donde la capacidad de significar no viene dada tanto por lo que se cuenta (visión temporal) sino por cómo se ordenan los elementos que hacen posible ese contar (visión espacial).

La escritura espacial, como fenómeno visual que tiene capacidad para significar por la disposición de los signos sobre el soporte, tiene también alguna servidumbre conceptual. Existe evidentemente otro espacio al menos, además del de la página. Se trata del espacio vivencial o referencial: el escritor hace percibir su obra como un espacio del texto y en el texto, pero el mundo al que la obra se refiere ya ha sido percibido a su vez según el mecanismo de la mirada ${ }^{18}$, un espacio bien delimitado por el campo visual, «quelque chose de vaguement rond, qui s'arrête très vite à gauche et à droite, et qui ne descend ni ne monte bien haut» ${ }^{19}$. Es decir, los mecanismos de percepción del mundo en el acto de contemplación de la realidad exterior, vuelven a repetirse luego en la percepción de la obra, y lo mismo que se puede decir de un paisaje, puede asimismo hacerse de una página escrita: «notre regard parcourt l'espace et nous donne ainsi l'illusion du relief et de la distance. C'est ainsi que nous construisons l'espace: avec un haut et un bas, une gauche et une droite, un devant et un derrière, un près et un loin»» ${ }^{19}$. Esta identificación o quizá simple paralelismo entre un concepto y otro no pone en entredicho la teoría de la escritura espacial; al contrario, si la escritura es efectivamente un acto de solidaridad

15. PEREC, G., Espèces d'espaces, Galilée, París, 1974, p. 18.

16. DENOËL, París, 1966.

17. MATHEWS, art. cit., p. 15.

18. Aquí «mirada» debe incluir la idea de percepción del mundo, a menos que se demuestre objetivamente que el escritor es un individuo al que no afecta la dependencia del entorno, en cuyo caso se demostraría la falsedad del principio orteguiano. El libro clave será Tentative d'épuisement d'un lieu parisien, C. Bourgois, París, 1975.

19. Espèces d'espaces, op. cit., p. 109 
respecto a todo aquello que no contiene, no es de extrañar que se reproduzcan abundantemente los mismos mecanismos de funcionamiento en lo espacial, con la única diferencia focal del que actúa en un preciso momento, es decir, el escritor enfrentado al mundo para construir la obra, el lector que percibe la obra con una estética renovada. En un caso se trata de la dependencia del mundo, hecho indefectible e irrefutable (es más, el escritor busca el grado máximo de identificación con esa realidad, digamos, objetal); en el otro se trata del consumidor de la obra ya terminada o no ${ }^{20}$, enfrentado al mundo de los signos que empiezan a significar siguiendo algunas pautas hasta ahora ignoradas.

El escritor escribe que está escribiendo, éste es el concepto de base para entender la escritura espacial. Desde «le texte se réfléchissant» de Mallarmé, la escritura se ha convertido en instrumento para definirse a sí misma, el medio coincide con el fin. A la hora de construir el espacio propio de la escritura perequiana aparece un fenómeno de gran importancia y trascendencia para lo que luego habrá de ser la trayectoria del escritor Perec: el recuerdo en su relación con lo escritural. En el caso de $W$ ou le souvenir d'enfance, libro donde se articula el concepto de escritura, el comienzo de la escritura debía coincidir con el comienzo de esa recuperación del recuerdo que supone para el escritor la verdadera vida, al liberarse de esa dependencia alienante del objeto, al conseguir aislarlo e instalarlo al margen de condicionamientos que siempre le oprimieron y que imposibilitaron de algún modo el escribir antes. La escritura es entonces un espacio descriptivo, es decir: la vida anterior cuyo recuerdo se halla muy deslavazado (por el paso del tiempo y por la fugacidad de aquellos acontecimientos), pues «si suprimiéramos de nuestra representación todas las imágenes y todos los recuerdos de objetos exteriores, suprimiríamos a la par el espacio» ${ }^{21}$. Esta particularidad del inicio en la actividad escritural de Perec se debe sin duda a la fusión de dos acontecimientos trascendentales, la coincidencia de dos momentos en un solo espacio que el libro consigue felizmente describir: «le projet d'écrire mon histoire s'est formé presque en même temps que mon projet d'écrire ${ }^{22}$; de esta manera, la escritura, al anular el tiempo de ese recuerdo, lo convierte en espacio donde aquel recuerdo (ausente ya de la mente del escritor) puede ser escritura, «souvenir de leur mort et l'affirmation de ma vie» ${ }^{23}$, la escritura sustituye al tiempo en el presente (puesto que sólo hay presente cuando se escribe, al carecer de dimensión temporal). Así es como la escritura funciona como espacio: por una lado, porque consigue eliminar la alienación temporal del recuerdo; por otro, porque consigue estabilizar el recuerdo mismo una vez que éste toma la forma de escritura.

El texto, producto final de la actividad escritural, es el delator por excelencia de la escritura, sobre todo y paradójicamente en los textos que evidencian sobre manera el hecho lingüístico, la actividad del lenguaje. En este caso podríamos decir que el texto utiliza el texto para hablar del texto, como en el ámbito del oulipismo, cuando la constricción se perfecciona hasta llegar a la máxima complejidad (cuando es un aparato en el sentido más profundo), el texto delata una apariencia formal in extremis, parece un cúmulo de ecuaciones, un cuaderno de claves: tal es el caso de Ulcérations $^{24}$ donde se exponen varias páginas con las secuencias permutacionales, o el de Alphabets $^{25}$ donde se exponen poema a poema las claves combinatorias debidamente descifradas.

20. Admitimos esta posibilidad puesto que así hay que entender toda la producción oulipiana, que permite al lector deconstruir la obra a partir de los mismos mecanismos que la construyeron (Oulipocrítica).

21. MACHADO, A., Los complementarios, Losada, Buenos Aires, 1957 (1968, 2.a), p. 50.

22. W ou le souvenir d'enfance, p. 41.

23. Ib., p. 59 .

24. Bibliothèque Oulipienne, n. ${ }^{\circ} 1,1974$.

25. Galilée, París, 1976. 
Estos ejemplos no hacen sino responder exactamente al concepto que el propio autor tenía de la escritura: «écrire: essayer méticuleusement de retenir quelque chose, de faire survivre quelque chose: arracher quelques bribes précises au vide qui se creuse, laisser, quelque part, un sillon, une trace, une marque ou quelques signes ${ }^{26}$. Y podemos llegar por el viaje entre bosques de citas y la intertextualidad a conclusiones aún más radicales o profundas. Lo que suponía el soporte de albergue para los signos de la escritura puede ahora trasladarse también al propio escritor: la página no será solamente un refugio para esos signos, será todo un acto de «écrire pour y chercher asile» ${ }^{27}$ por parte de Perec; la página con su escritura, la obra como unidad se constituirá en la figura de ese solar siempre ansiado donde poder instalarse. Sólo queda la escritura del propio autor para mostrarlo de manera definitiva, para demostrar también cómo se mueven los signos sobre la página, cómo se utilizan ordenadamente los recursos de distribución del texto, cómo en fin se produce la actividad escritural:

J'écris: j'habite ma feuille de papier, je l'investis, je le parcours.

Je suscite des blancs, des espaces (sauts dans le sens: discontinuités, passages, transitions).

J'écris

dans la

marge...

à la ligne. Je renvoie à une note en bas de page ${ }^{1}$

Je vais

Je change de feuille.

1. J'aime beaucoup les renvois en bas de page, même si je n'ai rien de particulier à y préciser.

Lo que se escribe a fin de cuentas es el texto, lo escrito es el texto porque, como ya señalamos, el texto es el producto de la escritura. También hemos afirmado que la escritura (en tanto que categoría nueva e independiente) funciona según criterios espaciales, en consecuencia, el texto producido habrá de ser también algo espacial, tanto si se construye con mecanismos espaciales como si delata un funcionamiento espacial.

Para algunos autores las causas de esta nueva manera de hacer se encuentran en la nueva dimensión que tiene la vida humana en relación con lo que sucede en el exterior: «l'homme d'aujourd'hui éprouve sa durée comme une «angoise», son intériorité comme une hantise ou une nausée; livré à l'«absurde» et au déchirement, il se rassure en projetant sa pensée sur les choses, en construisant des plans et des figures qui empruntent à l'espace des géomètres un peu de son assise et de sa stabilité ${ }^{29}$. Este desequilibrio acelerado de los acontecimientos de nuestra contemporaneidad es posiblemente la causa externa, el

26. Espèces d'espaces, p. 123.

27. PAWLIKOWSKA, E., «La colle bleue de G. Winckler», Littératures, n. ${ }^{\circ}$ 7, 1983, p. 80.

28. Espèces d'espaces, p. 19.

29. GENETTE, G., «Espace et langage», Figures I, Seuil, París, 1966, p. 101. Compruébese hasta qué punto esto es verdad si tenemos en cuenta algunas experiencias recientes; sin ir más lejos, en Perec, La Vie mode d'emploi (Hachette, París, 1978) es el ejemplo más claro de lo dicho y es la clave para entender lo que a continuación se dice: «aujourd'hui la littérature -la pensée- ne se dit plus qu'en termes de distance, d'horizon, d'univers, de paysage, de lieu, de site, de chemins et de demeure: figures naïves, mais caractéristiques, figures par excelence, où le langage s'espace afin que l'espace, en lui, devenu langage, se parle et s'écrive» (Genette, art. cit., p. 108) 
factor social de la encrucijada en que se encuentra la literatura y también la razón de que podamos expresarnos a partir de ahora en términos de escritura espacial del texto. Y efectivamente, la escritura como acto de solidaridad histórica no es sino el instrumento (y el fin) que servirá para reconducir ese «rapport de discorde» ${ }^{30}$ entre el hombre y la realidad exterior (las cosas, el cosmos).

Cuando el tiempo pierde su sentido porque lo que se pretende comunicar no es una historia (en el sentido tradicional-temporal o, al menos, no de esa manera), entonces las formas adquieren el protagonismo en la obra y empiezan a generar series de «cuadros descriptivos» que se asemejan bastante al terreno de la expresión plástica, no hay una linealidad secuencial constatable a primera vista, una etapa determinada no nos conduce obligadamente a otra (y que se pudiera prever este hecho), todo ocurre de manera distinta y el significado no viene dado ya precisamente por la ilación relacional que se establece en una cadena de elementos, sino por la ordenación distribucional de los componentes de aquel «cuadro», es decir, aparecen nuevos valores que ahora tienen significado pleno de manera que un componente aislado puede ya tener valor significativo como tal, sin tener que inscribirse en un sistema diacrónico o, al menos, no como hasta ahora venía ocurriendo ${ }^{31}$.

Descendiendo a casos más concretos, todo lo referente a la escritura (la escritura espacial) nos conduce siempre al texto. En Espèces d'espaces, libro que procura las claves del espacio que luego se desarrollará en otros libros (La Vie mode d'emploi principalmente), se nos da un concepto claro de lo que supone la actividad escritural respecto al texto; el espacio comienza con (las palabras) los signos trazados sobre la página en blanco, contraponiendo a la ausencia de color o de mancha toda una serie de trazos o manchas dibujadas:

J'écris...

J'écris: j'écris...

J'écris: «j'écris...»

J'écris que j'écris...

etc.

J'écris: je trace des mots sur une page.

Lettre à-lettre, un texte se forme, s'affirme, s'affermit, se fixe, se fige:

une ligne assez strictement $\mathrm{h}$

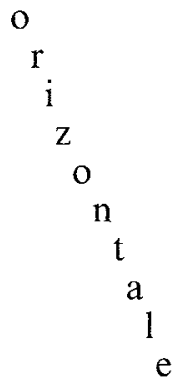

32

30. FRANK, J., «La forme spatiale dans la littérature moderne» Poétique, n. ${ }^{\circ}$ 10, 1972, p. 262. La tesis de este autor es que el universo histórico-temporal se ha transformado en un universo a-temporal (espacial) del mito y que por ello se da la llamada «forma espacial» de lo literario.

31. El caso más evidente sigue siendo La Vie mode d'emploi, a cuyo mecanismo se podrá acceder consultando, básicamente, los siguientes títulos: MAGNE, B., «Un puzzle de 100 pièces» (Magazine littéraire, n. ${ }^{\circ}$ 193, 1983); MAGNE, B., "Cinquième figure pour La Vie mode d'emploi» (Cahiers Georges Perec, n. ${ }^{\circ} 1,1985$ ); MAGNE, B., "La Vie mode d'emploi» itexto oulipiano?» (in Sobre literatura potencial, Univ. País Vasco, Zaragoza, 1987); sin olvidar por supuesto el título del propio autor en contribución al desentrañamiento de las claves de su libro: PEREC, G., «Quatre figures pour La Vie mode d'emploi») (in L'Arc, n. ${ }^{\circ} 76,1979$ ).

32. Espèces d'espaces, p. 17. Y sobre el productor de textos: «Assis à leur table, méditatifs et concentrés, les écrivains alignent des mots» (Ib., p. 23 el subrayado es nuestro). 
«C'est donc au départ l'espace du texte» ${ }^{33}$ también. Sobre qué o dónde hablar del espacio, pues sobre el espacio mismo (su espacio es la página y en ella se escribe sobre el espacio, el círculo se cierra). Este es el destino del escritor y de su escritura. De ahí el juego especular (continuo) al que asistimos a lo largo de La Vie mode d'emploi, como en el caso concreto de Valène: «il serait précisément en train de se peindre lui-même» (p. 291) que es la mejor metáfora (quizá ni siquiera una metáfora) para mostrar el mecanismo de los signos de la escritura.

La relación espacio-texto parece perfilarse entonces como el factor clave en el funcionamiento de la escritura espacial. Por un lado, el texto, para hacerse, debe ocupar un espacio, la página, el libro (el espacio dentro del texto, actitud «estática»); por otro lado, a medida que el texto se hace construye un espacio, se convierte en espacio (se va conformando el texto, actitud «dinámica»).

En el primer caso, se trata del horror vacui, la página-soporte-espacio está vacía y hay que llenarla, de ahí que los signos se distribuyan y que la escritura se plantee en muchas ocasiones como desafío, como reto o, sin embargo, como rutina.

En el segundo caso, entramos en un nudo de relaciones que tienen que ver con las disciplinas que se ocupan de la grafía como ciencia de transcripción del lenguaje; la definición radicalizada de esta servidumbre del texto respecto del espacio que ocupa, en el que se aloja, es el palimpsesto, crisol de textos, transformador y catalizador del destino escritural de un texto.

El futurible sin respuesta ad infinitum es «ia dónde va la literatura?», pregunta clave y permanente que posibilita la existencia de lo literario y de todo trabajo sobre la literatura. Se desciende al rasgo mínimo, al efecto más concreto, a la particularidad paradójicamente más activa: la escritura, traslación de la lengua, soporte a su vez del mensaje o mensaje mismo (todo un acto de reflexión definitivamente histórico). Lo extraño y evidente al mismo tiempo es que la escritura, para explicarse, recurre a la propia escritura. El escritor, al escribir, está dando la respuesta a la pregunta que su escritura plantea.

A partir de aquí el proceso se acelera enormemente, puesto que entramos ya en la consideración de lo espacial. Ya el aserto verba volant, scripta manent significa la perdurabilidad estable y atemporal de lo escrito, la expulsión del tiempo y la ampliación del campo a lo espacial: simplemente porque lo espacial se basa en la expansión, extensión, distribución, ordenación, etc., frente a la alineación, ilación, consecución, etc. típico de lo temporal. No es totalmente cierto que la escritura conlleve siempre el espacio $^{34}$, pero en cualquier caso es definitiva la relación escritura-espacio cuando se produce la reflexión sobre la escritura, cuando la escritura delata su mecanismo interno y se hace una categoría fuertemente autonímica.

El producto final de este sistema de tan complejo y al mismo tiempo de tan sencillo funcionamiento es un texto de carácter marcadamente espacial, «el espacio literario es el del texto; allí existe y allí tiene vigencia. Lo que no está en el texto es la realidad, lo irreductible a la escritura» ${ }^{35}$.

El universo perequiano se caracteriza por las relaciones que se establecen entre signos que constituyen o construyen la obra. Lo importante son las reglas seguidas para la puesta en relación de esos signos. Y la solución aportada por el autor a este problema

33. Entrevista de E. Pawlikowska a G. Perec, Littératures, n. ${ }^{\circ}$ 7, 1983, p. 72.

34. Esta afirmación deja de ser relativa en el instante en que nos referimos al concepto de escritura strictu sensu, puesto que el quehacer escritural es inseparable de una práctica de lo espacial.

35. GULLON, R., Espacio y novela, A. Bosch, Barcelona, 1980, p. 2. La cita es excesivamente tajante en el sentido perseguido por nosotros, pero sirve como guía de nuestra intención. 
es el funcionamiento espacial de todas estas relaciones: la obra funciona pues como un espacio. Pero ¿sobre qué apoyar el espacio literario de una obra? La respuesta viene directamente del hecho escritural: la escritura misma es el punto de referencia para la construcción del espacio de la obra y al mismo tiempo, haciéndose, la escritura se produce como espacio, se espacializa: ella estaba en el origen del espacio y ahora es el espacio el que regula la escritura. Una manera de vencer al tiempo. 